\title{
e-Migrinter
}

$8 \mid 2012$

Regards sur les migrations sud-asiatiques

\section{Howard S. Becker, Comment parler de la société. Artistes, écrivains, chercheurs et représentations sociales}

Caroline Rozenholc

\section{(2) OpenEdition \\ Journals}

Édition électronique

URL : https://journals.openedition.org/e-migrinter/673

DOI : 10.4000/e-migrinter.673

ISSN : 1961-9685

Éditeur

UMR 7301 - Migrinter

Édition imprimée

Date de publication : 4 avril 2012

Pagination : 144-146

ISSN : 1961-9685

Référence électronique

Caroline Rozenholc, «Howard S. Becker, Comment parler de la société. Artistes, écrivains, chercheurs et représentations sociales », e-Migrinter [En ligne], 8 | 2012, mis en ligne le, consulté le 20 mai 2021. URL : http://journals.openedition.org/e-migrinter/673; DOI : https://doi.org/10.4000/e-migrinter.673 


\section{Becker, Howard S. (2009) Comment parler de la société. Artistes, écrivains, chercheurs et représentations sociales, Paris, La Découverte, 316 p.}

\section{Caroline Rozenholc}

A vec Comment parler de la société, le sociologue américain Howard Becker, principalement connu en France pour son travail sur la "déviance », la consommation de marijuana et le jazz avec la traduction d'Outsiders en 1985, poursuit sa réflexion sur la pratique de la sociologie. Il y retrace plusieurs étapes d'un projet de recherche initié dans les années 1980 sur la transmission de l'information sociologique et les difficultés rencontrées par différents médias dans cette transmission. En effet, Howard Becker prend position dès la préface de cet ouvrage: les sciences sociales n'ont pas, pour lui, le monopole de la connaissance sur la société. Les romans, le théâtre, le cinéma et la photographie contiennent eux-aussi de nombreux éléments d'information sociétale et constituent, par conséquent, une source d'inspiration pour le travail sociologique.

Howard Becker livre ici deux parties de respectivement huit chapitres chacune dans lesquelles il s'applique à comparer les genres scientifiques et artistiques quant aux moyens déployés pour résoudre les questions de représentation de la réalité sociale. Les seize chapitres sont construits de sorte à se répondre sans devoir être toutefois lus de manière linéaire. Comment parler de la société. Artistes, écrivains, chercheurs et représentations sociales forme ainsi une collection d'articles où le propos de l'auteur se développe par la multiplication des exemples et des points de vue. "Idées", la première partie de l'ouvrage, rassemble des essais sur des sujets généraux, sur différents «mondes de représentation» alors qu'« Exemples », la deuxième partie du livre, est dévolue à l'analyse suivie de productions artistiques et scientifiques. Cela étant, le premier chapitre, "Parler de la société », est peut-être celui où le sociologue explicite le plus clairement sa perspective d'ensemble. Il y souligne, en effet, l'impossibilité de représenter totalement la réalité sociale; quand bien même les représentations que l'on en donne contiennent des observations pertinentes sur un point précis de fonctionnement de la société décrite. Dans ce sens, cartes et tableaux statistiques, romans et films sont autant de moyens de parler de la société dont $\mathrm{H}$. Becker cherche à tirer parti pour examiner, voire résoudre, les questions de représentation de la réalité sociale. La préoccupation de l'auteur est bien ici celle de la description et de l'ouverture à des solutions proposées dans des domaines qui, habituellement, ne communiquent pas : les statistiques avec la photographie documentaire ou les modèles mathématiques avec la littérature d'avant-garde. L'auteur cherche clairement de nouvelles perspectives d'analyse afin de proposer des solutions méthodologiques que les sciences sociales continuent souvent d'ignorer.

Dans la même veine, le deuxième chapitre, «Les représentations de la société. Des produits d'une organisation sociale », souligne la difficulté, en sociologie, à séparer les interprétations des faits. Dans son contexte social, tout fait ne suppose-t-il pas déjà des interprétations; et les scientifiques peuvent-ils procéder autrement qu'en transformant leurs matériaux qu'ils assemblent? Plus encore, puisque toute représentation est sélective et transformative, il faut chercher à comprendre, et à expliciter, ce qui préside à la transformation du matériel récolté. Cette question du choix des éléments retenus pour transcrire et communiquer sur la réalité de 
manière pertinente traverse d'ailleurs tout l'ouvrage et se retrouve au chapitre six dans «Condenser les détails ». Force est en effet de constater que la réalité sociale doit être largement réduite pour que puissent en émerger des représentations utiles et utilisables. Pour reprendre les termes mêmes de l'auteur, les représentations de la réalité sociale n'ont donc d'autres choix que de fabriquer «du peu à partir de beaucoup d'éléments» observés. S'il a le mérite de poser les questions et d'en décrire minutieusement les tenants et les aboutissants, la question des modalités de cette réduction, du passage de l'observation à la description pour parler de la société reste là peut-être entière.

Autre point important soulevé par H. Becker: celui de la charge du langage descriptif et de l'impossible transparence de ce dernier dans le domaine scientifique. Toutes les descriptions sont «chargées de théorie » (Kuhn 1970) et la méthode scientifique, elle-même, possède «une charge morale» significative. Pourtant, et c'est l'un des objets du chapitre huit, "Éthique de la représentation», les sociétés occidentales continuent d'entretenir l'idée de la neutralité du scientifique et de la science. Dans ces pages, H. Becker détaille alors comment les jugements moraux infusent jusqu'au choix des sujets abordés par les scientifiques, en particulier en sciences sociales (et par conséquent, si l'on poursuit son propos, également dans le domaine des études migratoires). Cette réflexion de l'auteur sur la transparence et l'ambiguité de la représentation, du contexte comme élément de compréhension de l'objet, déjà amorcée dans le chapitre trois, "Qui fait quoi ? ", à propos du travail photographique de l'américain Walker Evans, est ensuite reprise dans les pages consacrées à la sociologie visuelle (chapitre onze). Dans « Le travail des usagers" et "Standardisation et innovation» (chapitres quatre et cinq), c'est surtout aux usagers que le sociologue s'intéresse, aux efforts de lecture que ceux-ci doivent parfois fournir ; à la répartition entre fabricants et utilisateurs du travail de sélection, de transcription, de mise en forme et d'interprétation.

Dans la deuxième partie de son livre, Howard Becker aborde ensuite les fortunes diverses de plusieurs modèles mathématiques, «Paraboles, types idéaux et modèles mathématiques » (chapitre neuf), et les figures graphiques imaginées par certains sociologues pour rendre compte de réalités complexes telles que les systèmes de classes, «Figures: penser avec des dessins» (chapitre dix). Ces «Exemples» successifs illustrent tout à fait, à notre sens, le propos que l'auteur décline dans l'ensemble de son ouvrage, en insistant sur les possibilités créatives de ceux qui se donnent pour tâche de décrire certains pans de la société par des procédés et des formes «qui disent exactement ce qu'ils veulent dire » plutôt que de forcer leurs idées dans des formats standards (p. 196). Du chapitre onze "Sociologie visuelle, photographie documentaire et photojournalisme ", on retiendra que tous les modes de représentations - tout au moins ceux examinés dans ce livre - sont des constructions sociales. Les représentations ne peuvent donc avoir de sens fixe puisqu'elles baignent et sont issues, produites, dans des contextes sociaux toujours déterminants. Peut-être trouvera-ton là d'ailleurs, malgré la puissance d'évocation de la photographie - parfois beaucoup plus "parlante» qu'une longue description verbale -, la difficulté à justifier l'usage des matériels visuels dans les sciences sociales et leur relégation encore fréquente hors du champ scientifique.

Dans les cinq derniers chapitres de l'ouvrage, Howard Becker adopte une stratégie un peu différente pour aborder plusieurs œuvres classiques d'analyse sociales de manière suivie; qu'il s'agisse du théâtre de George Shaw, Caryl Churchill ou Wallace Shawn (chapitre douze), de la stratégie langagière qu'Erving Goffman (chapitre treize) adopte dans son travail comparatif ou 
de la prose de la romancière Jane Austen (chapitre quatorze). Dans le chapitre consacré à l'œuvre de Georges Perec (chapitre quinze), Howard Becker s'intéresse principalement aux procédés littéraires descriptifs dont use ce dernier. Il est vrai que l'atmosphère de Les choses (1968), Je me souviens (1978) ou encore Tentative d'épuisement d'un lieu parisien (1975) prime sur le récit et l'ambiance sur la linéarité du texte. Comme le note $\mathrm{H}$. Becker, on est, dans ces trois textes, très proche de la pratique des sociologues ou de la description ethnographique d'une culture, "avec ce que cela comporte de connaissances collectives et d'activités routinières » (p. 266). Il conclut d'ailleurs sa réflexion sur les travaux de Perec par une note rapide, mais tout à fait intéressante, en soulignant que l'ensemble des descriptions sociales est structuré à la fois par le désir de montrer et par celui d'expliquer. De fait, l'analyse sociologique s'en trouve également mise en tension entre souci descriptif et volonté explicative.
Un dernier chapitre sur l'œuvre d'Italo Calvino (chapitre seize) vient clore cet ouvrage par quelques remarques particulièrement éclairantes quant à l'entreprise de Comment parler de la société. Artistes, écrivains, chercheurs et représentations sociales. L'auteur y relève non seulement comment les faits sont mis en forme par nos idées et combien il est difficile de voir ce que nous ne sommes pas préparer à observer, mais également qu'aucun phénomène ne peut se réduire à une explication, à un seul type de description. Si, là encore, l'auteur insiste sur la multitude de possibilités que nous offre le monde plutôt que sur l'existence de méthodes idéales pour le décrire, sur les possibilités souvent ignorées d'exploration et de représentation de la société, qu'il nous pardonne alors d'avoir synthétisé un peu dans le désordre les mailles de son ouvrage.

Caroline Rozenholc Docteure en Géographie Migrinter - CRFJ caroline.rozenholc@,univ-poitiers.fr 